\title{
Hiérarchisation de l'impact morphologique d'événements climatiques contrastés sur la plage de Grand'Rivière (Martinique)
}

\author{
K. Bosser ${ }^{1}$, F. Levoy ${ }^{2}$, J.C. Flageollet ${ }^{3}$ \\ ${ }^{1}$ Post-Doct., U.S Geological Survey, Menlo Park, Californie. \\ ${ }^{2}$ Maître de Conférence, Université de Caen, Unité de \\ Morphodynamique Continentale et Côtière. \\ ${ }^{3}$ Professeur Emérite, Université Louis Pasteur de Strasbourg.
}

\section{Résumé.}

Aux Antilles, les cyclones et les épisodes de renforcement des alizés sont les principaux responsables de l'érosion côtière. Une hiérarchisation de ces tempêtes est proposée en utilisant un modèle de transport sédimentaire. La comparaison des taux de transports journaliers montre que les houles cycloniques sont les plus érosives comparées aux alizés. Mais du fait de leur durée et de leur fréquence, les renforcements d'alizés d'intensité modérée et forte mobilisent de plus grands volumes de sédiments. Ils peuvent être supérieurs de 10 à $40 \%$ par rapport à ceux déplacés par les cyclones. Par contre, pour des intensités extrêmes, les taux de sédiments mobilisés par les cyclones représentent cinq fois ceux observés lors d'une période d'alizés modérés. Ce sont ces événements majeurs qui induisent une évolution significative de la plage et du trait de côte à long terme.

\section{Summary.}

In the West Indies, tropical storms, hurricanes and Trade Wind intensification events are the main agents causing erosion. The relative importance of each agent is determined in this paper using a cross-shore sediment transport model. This model predicts that cross-shore sediment transport rates during tropical storms and hurricanes are larger than during Trade Wind events. However, because Trade Wind events are more frequent and last longer, they cause $10 \%$ and $40 \%$ more erosion, respectively, than moderate and strong hurricanes. But, for extreme intensities, net cross-shore sediment transport due to hurricanes is about five times the transport predicted for a moderate Trade Wind event. Those large events may have long term consequences on this coast.

\section{Introduction}

Les houles de tempêtes sont les principaux facteurs responsables de l'érosion côtière dans les milieux tropicaux microtidaux. Comprendre les changements induits par ces tempêtes est d'un intérêt capital pour améliorer la prédiction à court et long terme de l'évolution du trait de côte. Cela implique, au préalable, une 
bonne connaissance des caractéristiques hydrodynamiques lors de ces conditions à savoir, la hauteur des houles, les périodes, la direction de provenance, la fréquence d'apparition et la durée de ces événements. Il est souvent difficile de mesurer sur le terrain le transport associé aux tempêtes, notamment d'intensité extrême. Dans le but d'accroître nos connaissances, le modèle de transport sédimentaire UNIBEST (Delft Hydraulics, 1994), utilisant des mesures in situ acquises entre mai 1995 et avril 1997 à Grand'Rivière en Martinique, a été utilisé. Le but est d'établir une hiérarchie des conditions hydrodynamiques contribuant à l'évolution de ce littoral, afin de mieux prévoir l'impact des cyclones et des tempêtes tropicales sur cette côte.

Dans une première partie, les scénarii de tempêtes sélectionnés pour les simulations sont définis. Différentes directions de provenance de houles et d'intensités ont été prises en compte. Ensuite, les résultats des simulations des taux de transports sédimentaires et des volumes mobilisés sont présentés. Pour finir, l'impact d'un cyclone de très forte énergie a été simulé. Il s'agit du cyclone DAVID, ayant causé de sévères dommages lors de son passage au nord de la Martinique en Août 1979. Son impact morphologique a été comparé aux fortes tempêtes observées entre 1995 et 1997.

\section{Le site étudié}

Le site étudié, Grand'Rivière, est localisé au nord de la Martinique (Fig. 1). L'érosion côtière, comprise entre 0,5 et $2,5 \mathrm{~m} . \mathrm{an}^{-1}$, est favorisée par les fortes houles et la nature pyroclastique des sédiments (Bosser, 1998). La morphologie de la côte est caractérisée par deux plages, celle de l'anse Bagasse et celle de l'anse Morne Rouge, localisées aux extrémités est et ouest d'un cône alluvial. La plage de l'anse Bagasse (Fig. 1) est étroite, limitée par des falaises d'environ $20 \mathrm{~m}$ de hauteur. La marée est de faible amplitude, en moyenne inférieure à $0,30 \mathrm{~m}$ (Kjerfve, 1981). Le climat de houle est caractérisé par des hauteurs annuelles moyennes au large de l'ordre de 1,20 à $1,60 \mathrm{~m}$, avec une période comprise entre 6 et $10 \mathrm{~s}$. Les fortes houles peuvent avoir deux origines différentes : les cyclones et les épisodes de renforcement des alizés (Bosser et al, 2000a). Ces derniers se produisent lors du déplacement vers le nord des anticyclones sur l'Atlantique. Au cours des années 1995 à 1997, de fréquents épisodes d'intensification des alizés et plusieurs tempêtes tropicales et cyclones ont été observés. En moyenne, la hauteur significative maximale de houle atteinte lors des épisodes d'intensification des alizés est comprise entre 2 et 4,5 m. Pour les tempêtes tropicales et les cyclones, elle varie entre 3 et $7 \mathrm{~m}$ dans cette région (Bleuse et al. 1995). Même s'ils atteignent des hauteurs parfois similaires, la durée des épisodes de renforcements des alizés est généralement plus longue que celle des cyclones, d'où un risque d'érosion accru pour la côte lors de ces évémements (Bosser \& Levoy 1998 ; Bosser et al., 2000b). Ces deux types de tempêtes ont donc été analysés séparément dans cette étude afin de comparer leurs impacts morpho-sédimentaires spécifiques. 


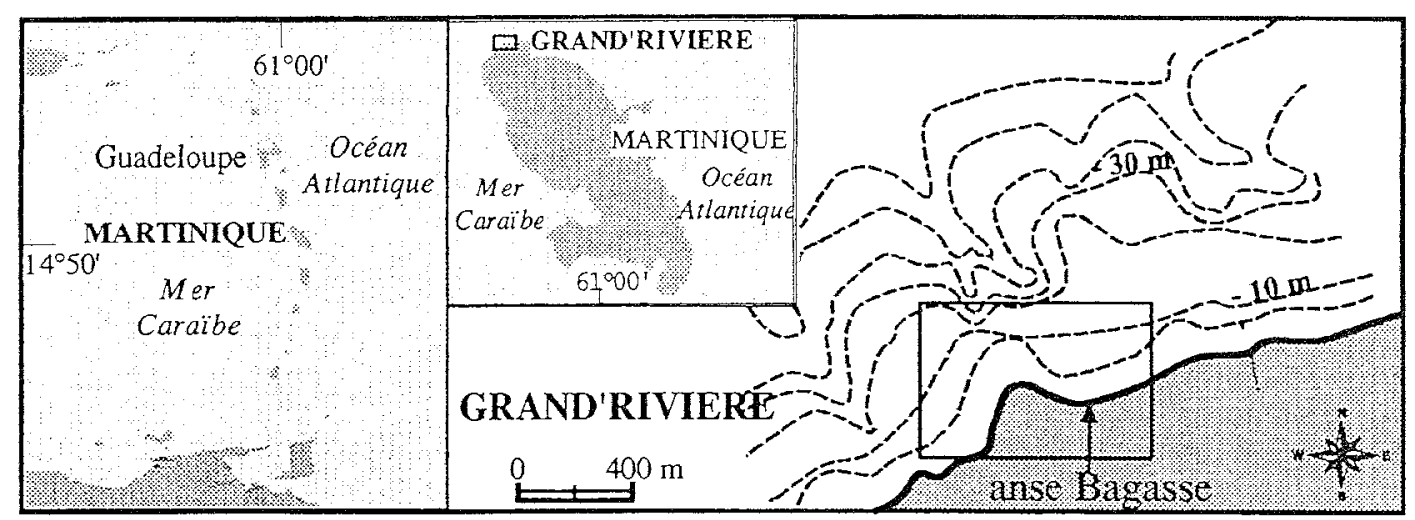

Fig. 1. Localisation du site étudié, Grand'Rivière en Martinique.

Fig. 1 : Location of the study site, Grand'Rivière, Martinique Island.

\section{Description des modèles et validation}

Deux modèles numériques ont été utilisés afin de prévoir le transport sédimentaire perpendiculaire à la côte, en période de tempête, sur la plage de l'anse Bagasse. Le premier, HISWA (Delft Hydraulics, 1995), calcule la propagation de la houle sur l'avant-côte. Le second, UNBBEST (Delft Hydraulics, 1994), calcule l'évolution du profil induit par le changement des caractéristiques de l'agitation à la côte. Pour simuler l'évolution du profil de plage, il est nécessaire de connaitre la déformation de la houle à l'approche du littoral. Celle-ci est calculée dans les faibles profondeurs avec HISWA à partir des données bathymétriques et des hauteurs de houle au large. Les conditions de houle aux limites de la zone d'étude sont issues de mesures réalisées à l'aide d'un houlographe directionnel mouillé à $60 \mathrm{~m}$ de profondeur au NNE de Grand'Rivière. Les données recueillies sont utilisées comme conditions initiales pour le modèle HISW A. La grille bathymétrique est issue de levés réalisés en juillet 1995, octobre 1996 et mai 1997. Les paramètres et coefficients utilisés dans le modèle, sa validationin ainsi que les tests de sensibilité réalisés sont décrits dans Bosser et al, 2000a.

Le modèle UNIBEST utilise d'une part, des données topographiques initiales de conditions de pré-tempête et d'autre part, des informations concernant l'agitation sur la zone étudiée. Ce modèle détermine l'évolution de la morphologie transversale à partir des courants «cross-shore » simulés en période de tempête. Le profil bathymétrique utilisé dans les simulations est situé au milieu de la plage de l'anse Bagasse. Sa longueur, englobant la plage et l'avant-côte est de $1200 \mathrm{~m}$. La formule de transport sédimentaire qui simule au mieux les conditions mesurées à Grand'Rivière est celle de Bailard. La comparaison entre les données de terrain et les résultats des modèles apparaît satisfaisante, permettant d'envisager la simulation de divers scénarii hydrodynamiques (Bosser et al, 2000a).

\section{Sélection des scénarii de houle}


Les scénarii de houle pris en considération ont été sélectionnés à partir des données de houle mesurées entre avril 1995 et mai 1997 et à partir d'exemples pris dans la littérature pour les événements extrêmes (Pagney, 1992; Bleuse et al., 1995 ; Durand, 1996).

Deux conditions de houle ont été prises en considération: (i) les houles venant des épisodes d'intensification des alizés (15\% des observations), (ii) les houles cycloniques ( $5 \%$ des observations). Pour ces deux types, trois classes de hauteurs de houle ont été définies dans le tableau 1. Pour les événements d'intensité extrême, les houles issues des alizés sont limitées à 2,9 m à cause du fetch, alors que la hauteur des houles induite par des cyclones extrêmes peut atteindre environ 3,5 m près de la côte de Grand'Rivière. Les surcotes ont été calculées à partir de la pression barométrique (Maul et al,. 1995). Les durées moyennes choisies pour les simulations, établies d'après les données de terrain et la littérature, sont décrites dans le tableau 1. La définition des durées et du nombre d'épisodes de renforcement des alizés à partir des données de terrain a été faite de la façon suivante. Une hauteur significative de la houle supérieure à 1,5 $\mathrm{m}$ a été retenue, valeur à partir de laquelle l'érosion de la plage de l'anse Bagasse a été clairement mesurée (Bosser et al, 2000a). La durée d'une tempête esst ensuite définie comme le nombre de jours durant lequel les hauteurs de houle ont dépassé 1,5 $\mathrm{m}$. Trois directions de houle ont été étudiées (E, NE et ESE), basées sur les directions de houle de tempête mesurées au houlographe de Grand'Rivière.

A partir des scénarii hydrodynamiques définis, le taux de transport sédimentaire journalier associé à chaque tempête a été simulé afin de comparer leur débit respectif. Ensuite, en tenant compte de la durée de chaque événement, les volumes sédimentaires mobilisés ont été calculés.

\section{Résultats}

Le tableau 1 montre une grande variabilité des transports sédimentaires «crossshore » journaliers, calculés pour la plage de l'anse Bagasse pour chaque scénario sélectionné. Le modèle prédit un transport sédimentaire globalement plus important pour les cyclones d'intensité modérée $\left(26\right.$ à $45 \mathrm{~m}^{3} \cdot \mathrm{m}^{-1}$.jour $\left.\mathrm{r}^{-1}\right)$ et forte $(57$ à $82 \mathrm{~m}^{3} \cdot \mathrm{m}^{-1} \cdot$ jour $\left.{ }^{-1}\right)$ que pour les alizés de même intensité $\left(16\right.$ à $32 \mathrm{~m}^{3} \cdot \mathrm{m}^{-1}$.jour ${ }^{-1}$ et 20 à $54 \mathrm{~m}^{3} \cdot \mathrm{m}^{-1} \cdot$ jour $^{-1}$ ).

Si l'on considère les volumes mobilisés à l'échelle de l'événement (Fig. 2), il apparaît que les épisodes de renforcement des alizés d'intensité modérée et fortes ont un impact plus prononcé que les cyclones de même intensité sur l'évolution morphodynamique de la plage de Grand'Rivière (volumes supérieurs de 10 à 40 $\%$ ). La raison principale est la durée particulièrement longue des épisodes de renforcement des alizés, environ 7 jours en moyenne, comparée à celle d'un cyclone, environ 2,5 jours.

Par contre, pour des intensités extrêmes, les volumes sédimentaires mobilisés par les cyclones sont, en moyenne, deux fois plus importants que ceux mobilisés par les alizés les plus forts. Ceci peut s'expliquer par la différence de hauteur de houle atteinte par ces deux types de phénomènes. 


\begin{tabular}{|c|c|c|c|c|c|}
\hline $\begin{array}{l}\text { Conditions de } \\
\text { houle }\end{array}$ & $\begin{array}{l}\text { Hs près de la } \\
\text { côte }(m) \text { pour } \\
\text { différents } \\
\text { événements }\end{array}$ & $\begin{array}{l}\text { Surcote } \\
\text { moyenne } \\
* *(\mathrm{~m})\end{array}$ & $\begin{array}{c}\text { Durée } \\
\text { moyenne } \\
\text { (jours) }\end{array}$ & $\begin{array}{c}\text { Directions } \\
\text { de houle }\end{array}$ & $\begin{array}{c}\text { Transport } \\
\text { sédimentaire } \\
\left(\mathrm{m}^{3} \cdot \mathrm{m}^{-1} \cdot \mathrm{j}^{-1}\right)\end{array}$ \\
\hline \multirow{3}{*}{$\begin{array}{l}\text { Episode de } \\
\text { renforcement } \\
\text { des alizés }\end{array}$} & $\begin{array}{c}\text { entre } 1,5 \text { et } 2,5 \\
\text { intensité } \\
\text { modérée }\end{array}$ & 0,1 & 2,75 & $\begin{array}{c}\text { NE } \\
\mathrm{E} \\
\mathrm{ESE} \\
\end{array}$ & $\begin{array}{l}32 \\
28 \\
16 \\
\end{array}$ \\
\hline & $\begin{array}{l}\text { entre } 2,5 \text { et } 2,9 \\
\text { intensité forte }\end{array}$ & 0,15 & 3,5 & $\begin{array}{c}\mathrm{NE} \\
\mathrm{E} \\
\mathrm{ESE} \\
\end{array}$ & $\begin{array}{l}54 \\
45 \\
20 \\
\end{array}$ \\
\hline & $\begin{array}{c}>2,9 \\
\text { intensité } \\
\text { extrême }\end{array}$ & 0,2 & 7 & $\begin{array}{c}\mathrm{NE} \\
\mathrm{E} \\
\mathrm{ESE} \\
\end{array}$ & $\begin{array}{l}70 \\
60 \\
32 \\
\end{array}$ \\
\hline \multirow{3}{*}{ Cyclone } & $\begin{array}{l}\text { entre } 1,5 \text { et } 2,5 \\
\text { intensité } \\
\text { modérée } \\
\text { categorie } 1 *\end{array}$ & 0,3 & 1,5 & $\begin{array}{c}\text { NE } \\
E \\
E S E\end{array}$ & $\begin{array}{l}45 \\
38 \\
26\end{array}$ \\
\hline & $\begin{array}{l}\text { entre } 2,5 \text { et } 3,5 \\
\text { intensité forte } \\
\text { categorie } 2-3 *\end{array}$ & 0,7 & 2 & $\begin{array}{c}\mathrm{NE} \\
\mathrm{E} \\
\mathrm{ESE}\end{array}$ & $\begin{array}{l}82 \\
60 \\
57\end{array}$ \\
\hline & $\begin{array}{l}>3,5 \text { intensité } \\
\text { extrême } \\
\text { categories } 4.5^{*}\end{array}$ & 1,1 & 3 & $\begin{array}{c}\text { NE } \\
E \\
\text { ESE }\end{array}$ & $\begin{array}{l}277 \\
224 \\
200\end{array}$ \\
\hline
\end{tabular}

* basé sur l'échelle de Saffir-Simpson (SimpSON, 1971).

** au-dessus du niveau local de référence $(-0,54 \mathrm{~m})$.

Tableau 1: Hiérarchisation des taux de transport sédimentaire calculés pour des événements climatiques d'intensités et de directions variées (plage Bagasse, Martinique).

Table 1: Hierarchical organization of daily sediment transport rates calculated for climatic events of various intensities and directions (Bagasse beach, Martinique island).

En effet, les hauteurs significatives maximales de houle près de la côte associées à des alizés extrêmes ne peuvent excéder $2,90 \mathrm{~m}$ à cause du fetch limité. Par contre, les hauteurs générées par les cyclones extrêmes peuvent être supérieures à 3,5 m.

Ces simulations montrent également que les houles venant de l'E et du NE sont plus érosives que celles venant de l'ESE pour la côte étudiée. Ceci s'explique par une dissipation d'énergie plus importante des houles venant de l'E et de l'ESE sur le plateau insulaire bordant Grand'Rivière par rapport à celles provenant du NE.

Une comparaison entre trois événements de nature et intensité différentes est proposée afin de montrer leur impact sédimentaire respectif sur la côte (Fig. 3). Il s'agit d'une simulation du cyclone extrême DAVID (1979), qui a provoqué de très importants dégâts sur la côte étudiée, comparé au cyclone modéré LUIS (1995) et à l'épisode de renforcement des alizés d'octobre 1996. Un facteur 5 apparaît entre le transport induit par le cyclone DAVID et les deux autres d'événements (Fig. 3), 
ce qui illustre le fort pouvoir érosif des cyclones extrêmes. L'impact à long terme sur la position du trait de côte de tels cyclones a été confirmé par l'étude de photographies aériennes sur 50 ans (Bosser, 1998).

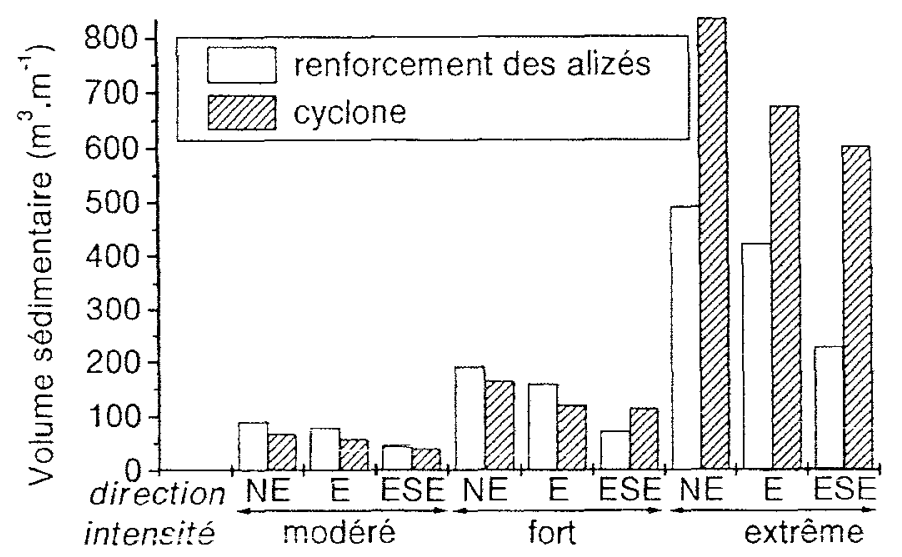

Figure 2. Volumes sédimentaires caiculés pour des événements climatiques d'intensité variée (plage Bagâsse à Grand'Rivière).

Figure 2 : Sediment transport volumes calculated for various climatic events (Bagasse beach, Grand'Rivière, Martinique island).

\section{$\underline{\text { 6. Discussion }}$}

Ces simulations, basées sur des mesures de terrain et la littérature, permettent de hiérarchiser les taux de transports sédimentaires «cross-shore induits par des tempêtes tropicales de niveaux d'énergie et de directions variés. Les résultats numériques obtenus pour des cyclones d'intensité forte (catégories 2 et 3) frappant la Martinique sont semblables à ceux observés pour d'autres sites présentés dans la littérature à partir d'investigations sur le terrain. Ainsi, Morton et al. (1994) ont étudié l'érosion associée au cyclone ALICIA (catégorie 3) sur les côtes sud-est du Texas. Celui-ci a érodé un volume de sédiments compris entre $51 \mathrm{~m}^{3} \cdot \mathrm{m}^{-1}$.jour ${ }^{-1}$ et $73 \mathrm{~m}^{3} \cdot \mathrm{m}^{-1}$.jour ${ }^{-1}$, ce qui correspond à l'ordre de grandeur de nos résultats pour une même intensité $\left(57\right.$ à $\left.82 \mathrm{~m}^{3} \cdot \mathrm{m}^{-1} \cdot \mathrm{jour}^{-1}\right)$. Cependant, pour des intensités extrêmes (catégories 4-5), les résultats de nos simulations sont légèrement différents des mesures de terrain relevées dans la littérature. Dingler \& Reiss (1995) montrent ainsi qu'au cours du cyclone ANDREW (catégorie 4), passé à moins de $50 \mathrm{~km}$ des Mles Dernières (Louisiane), une perte sédimentaire de $81 \mathrm{~m}^{3} \cdot \mathrm{m}^{-1}$.jour ${ }^{-1}$ à $92 \mathrm{~m}^{3} \cdot \mathrm{m}^{-}$ ${ }^{1}$.jour ${ }^{-1}$ a été observée. Ainsi, il semblerait que le modèle numérique utilisé dans cette étude surestime l'érosion induite par les cyclones extrêmes au nord de la Martinique $\left(220 \mathrm{~m}^{3} \cdot \mathrm{m}^{-1} \cdot \mathrm{jour}^{-1}\right)$. Cependant, il faut noter que la comparaison des taux de transport sédimentaire entre des endroits différents est délicate en raison de la spécificité morphologique de chaque site. Par ailleurs, le modèle UNIBEST limite l'évolution du profil transversal de la plage et de l'avant-côte de façon, semble-t-il, prématurée. 


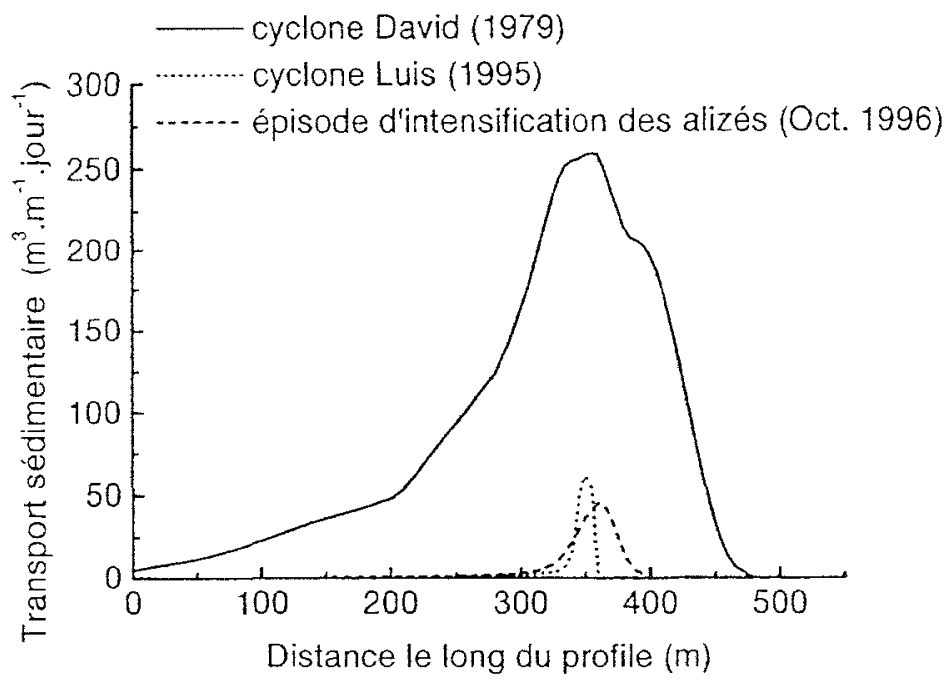

Figure. 3 : Comparaison du taux de transport sédimentaire induit par des houles extrêmes (DAVD, 1979), des houles cycloniques modérées (LUIS, 1995) et des houles issues d'un épisode de renforcement des alizés modéré (Oct. 1996).

Figure. 3 : Comparison between sediment transport induced by extreme cyclonic waves (DAVID, 1979), moderate cyclonic waves (LUIS, 1995) and waves induced by a moderate intensification of the Trade Winds (Oct. 1996).

En effet, la profondeur maximale de remaniement sédimentaire apparait être située vers $-10 \mathrm{~m}$ lors des simulations de cyclones d'intensités extrêmes. Durand (1996), en étudiant l'impact d'un tel cyclone sur les fonds sédimentaires de cette zone a mis en évidence, la présence de mégarides de tempêtes à une profondeur d'environ $-20 \mathrm{~m}$, positionnant, de fait, la limite d'action des houles extrêmes beaucoup plus profonde que celle mise en évidence sur les simulations.

\section{Conclusion}

Basées sur des mesures de terrain (mai 1995 - avril 1997), des simulations numériques du transport sédimentaire «cross-shore » lors de tempêtes et cyclones tropicaux ont été effectuées afin d'examiner l'évolution de la principale plage de Grand'Rivière en Martinique. Une hiérarchie de l'impact respectif des cyclones et des épisodes de renforcement des alizés sur l'érosion de la plage et de son prolongement subtidal a été établie selon l'intensité, la direction et la durée de ces événements. Cette étude met en évidence l'impact plus important des épisodes de renforcement des alizés d'intensité modéré et forte comparé aux cyclones de même intensité, moins fréquents et d'une durée plus courte. Les cyclones extrêmes, quant à eux, capables de mobiliser deux fois plus de sédiments que les alizés extrêmes, ont des conséquences importantes sur le recul du trait de côte de Grand'Rivière et son évolution sur le long terme. 


\section{Remerciements}

Nous tenons à remercier le Conseil Général de la Martinique pour le support financier qu'il a apporté à cette étude.

\section{Références}

Bleuse, P., A. Soulan, P. Frayssinet \& N. Bleuse (1995): 1995, l'année de tous les cyclones - METEO FRANCE (eds), 39 pp.

BOSSER, K. (1998) : Evolution morphodynamique du littoral Nord martiniquais soumis à l'aléa cyclonique - L'exemple de Grand'Rivière. - Thèse de I'Université Louis Pasteur de Strasbourg (PhD thesis), France, 280 pp.

Bosser, K. \& F. LEVOY (1998): Impact hydro-sédimentaire des périodes de renforcement des alizés sur une plage de la côte nord de la Martinigue - Actes des $V^{\text {èmes }}$ journées nationales Génie Côtier - Génie Civil, Toulon 13 au 15 mai 1998: 111-119.

Bosser, K., F. Levoy, J.C. Flageollet, O. Monfort \& H. Rousset (2000a) : Trade wind intensification and hurricanes on a Carribean beach, Martinique Island: a comparison of their morphological impacts from field experiments and numerical simulations. - Journal of Coastal Research (en cours de publication).

Bosser, K., F. Levoy, J.C. Flageollet (2000b) : Evolution morphodynamique du littoral nord Martiniquais soumis à l'aléa cyclonique - Géomorphologie (accepté pour publication).

DELFT HYDRAUliCS (1994): UNIBEST Theorical reference manual document, Programme Manual : 39 pp.

DELFT HYDRAULICS (1995): HISWA user manual, Prediction of stationary shortcrested waves in shallow water with ambient currents, version 110-43, $11 \mathrm{pp}$.

DINGLER, J.R \& T. REISS (1995): Beach erosion on Trinity Island, Louisiana caused by Hurricane Andrew - Journal of Coastal Research. Special Issue. 21 : 254-264.

DURAND, F. (1996): Hydrodynamique sédimentaire sur le plateau insulaire de la Martinique - Thèse Université Bordeaux I, 214 pp.

KJerfye, B. (1981): Tides of the Caribbean Sea. - Journal of Geophysical Research. C5. $86: 4243-4247$.

Maul G.A, M.D. Hendry \& P.A. Pirazzoli (1995): Sea level, tides and tsunami. In: Small Island Marine Science and Sustainable Development Coastal and Estuarine Studies, AGU Edition, 51 : 83-119.

Morton, R.A , J.G. PaINe \& J.C. Gibeaut (1994) : Stages and duration of poststorm beach recovery, Southeastern texas coast, U.S.A. - Journal of Coastal Research. 10, 4:884-908.

PAGNEY, F. (1992): Les Antilles françaises, des îles sous triple hypothèque: sismique, volcanique et cyclonique. - Historiens-Géographes. 335 : 361-377.

SIMPSON, R.H. (1971): A proposed scale for ranking hurricanes by intensity Minutes of the Eighth NOAA, NWS Hurricane Conference, Miami, FL. 